\title{
Biozide in Gebäudefassaden - ökotoxikologische Effekte, Auswaschung und Belastungsabschätzung für Gewässer
}

\author{
Michael Burkhardt • Marion Junghans • Steffen Zuleeg • Ute Schoknecht • Xolelwa Lamani · Kai Bester • \\ Roger Vonbank · Hans Simmler • Markus Boller
}

Erhalten: 13. Juli 2008/Akzeptiert: 31. Oktober 2008/Online veröffentlicht: 13. Januar 2009

(C) Springer-Verlag 2009

Zusammenfassung Hintergrund, Ziel und Zweck Die Belastung von Fließgewässern durch organische Mikroverunreinigungen wirft häufig die Frage der Quellenzuordnung auf. Baumaterialien als Quellen problematischer Spurenstoffe finden in der Gewässerbeurteilung bisher wenig Beachtung, obwohl bereits heute biozide Wirkstoffe aus Materialschutzanwendungen zu einer Belastung des Regen-

Zusätzliche Information ist in der online Version dieses Beitrags (doi: 10.1007/s12302-008-0033-1) enthalten.

Verantwortlicher Herausgeber: Walter Giger

M. Burkhardt $(\bowtie) \cdot$ M. Junghans $\cdot$ S. Zuleeg $\cdot$ M. Boller Eawag - Das Wasserforschungs-Institut des ETH-Bereichs, Abteilung Siedlungswasserwirtschaft,

Überlandstrasse 133, 8600 Dübendorf, Schweiz

E-Mail: michael.burkhardt@eawag.ch

M. Junghans

neu: BMG Engineering AG,

Ifangstrasse 11, 8952 Schlieren, Schweiz

U. Schoknecht

Bundesanstalt für Materialforschung und -prüfung (BAM),

Fachgruppe IV.1 ,Biologie im Umwelt- und Materialschutz,

Unter den Eichen 87, 12205 Berlin, Deutschland

X. Lamani $\cdot$ K. Bester

Universität Duisburg-Essen, Institut für Umweltanalytik,

Universitätsstrasse 15, 45141 Essen, Deutschland

\section{K. Bester}

neu: Universität Aalborg, Department of Biotechnology,

Chemistry and Environmental Engineering,

Sohngaardsholmsvej 57, 9000 Aalborg, Dänemark

R. Vonbank $\cdot$ H. Simmler

Eidgenössische Materialprüfungs- und Forschungsanstalt (Empa),

Abteilung Bautechnologien,

Überlandstrasse 129, 8600 Dübendorf, Schweiz wasserabflusses führen. In der vorliegenden Untersuchung wurden (1) die ökotoxikologischen Kenngrößen für typische Biozide in Fassadenbeschichtungen ermittelt, (2) die Auswaschung von besonders problematischen Bioziden aus Fassaden quantifiziert und (3) das Belastungsrisiko für Gewässer mittels Stofftransportmodellierung abgeschätzt.

Methoden Acht Biozide wurden untersucht, die in kunstharzgebundenen Fassadenbeschichtungen verwendet werden. Dazu gehören auch Wirkstoffe, deren Umwelteintrag bisher eher der Landwirtschaft zugerechnet wurde, z. B. Diuron, Carbendazim und Terbutryn. Für jedes Biozid wurden ökotoxikologische Qualitätskriterien für aquatische Organismen bestimmt. Die Auswaschung von vier Bioziden aus einer verputzten Fassade wurde im Laborversuch unter Berücksichtigung von UV-Bestrahlung untersucht, wobei die Temperatur variiert wurde. Über 80 Beregnungsintervalle in 28 Tagen wurden Proben des Fassadenabflusses entnommen, die Wirkstoffkonzentrationen gemessen und die ausgewaschenen Wirkstoffmengen berechnet. Basierend auf einem Austragsszenario wurde das mögliche Belastungsrisiko im Gewässer durch das Biozid Cybutryn mit einer Stofftransportmodellierung abgeschätzt. Die ermittelten wirkstoffspezifischen Qualitätskriterien und die Auswaschcharakteristik wurden darin berücksichtigt.

Ergebnisse Die akuten und chronischen Kriterien sowie vorhergesagten Konzentrationen ohne Effekt weisen auf eine hohe Ökotoxizität der betrachteten Biozide hin. Die Ergebnisse zum Auswaschungsverhalten von vier Bioziden (Diuron, Terbutryn, Cybutryn und Carbendazim) zeigen, dass die Wirkstoffe im Fassadenabfluss vorkommen und die Belastung unter den gewählten experimentellen Bedingungen exponentiell abnimmt. Dabei führt eine Temperaturerhöhung wieder zu einem Konzentrationsanstieg. Die ausgewaschenen Stoffmengen liegen zwischen 7\% und $29 \%$. Bereits innerhalb der ersten 15 min wurde mehr als 
die Hälfte der gesamten Stoffmenge während der 60 min Beregnungsdauer ausgewaschen. Die Stofftransportmodellierung zum Eintrag des Biozids Cybutryn aus Fassaden ins Gewässer deutet auf ein hohes Belastungspotential für kleinere Gewässer hin.

Diskussion Die Ergebnisse zur Auswaschung von Bioziden, deren potentielle ökotoxische Effekte und Stabilität in der Umwelt zeigen, dass von einigen Wirkstoffen für Gewässer ein hohes potentielles Belastungsrisiko, von anderen aber nur ein geringes Risiko ausgehen dürfte. Belastetes Fassadenwasser muss für einige Biozide sehr deutlich durch unbelastetes Regenwasser verdünnt werden, damit die Qualitätskriterien im Gewässer eingehalten werden. Diuron und Carbendazim werden auch in anderen Materialschutzanwendungen verwendet oder in der Landwirtschaft ausgebracht. Cybutryn wird oft als Antifouling-Wirkstoff in Bootsanstrichen eingesetzt, so dass eine Belastung im Gewässer erst unter Berücksichtigung aller Eintragswege beurteilt werden kann.

Schlussfolgerungen Kritische Konzentrationsbereiche im Fassadenabfluss sind an neuen Gebäudefassaden zu erwarten, in der Regel vor allem an wärmegedämmten Fassaden. Angesichts der niedrigen vorhergesagten Konzentrationen ohne Effekt im Bereich von wenigen $\mathrm{ng} / \mathrm{L}$ und den hohen Anwendungsmengen in Fassaden sollte die Auswaschung und das Umweltverhalten wirkstoffspezifisch abgeschätzt werden. Maßnahmen an der Quelle und die jeweils nachhaltigste Regenwasserentsorgung sollten evaluiert werden.

Empfehlungen und Ausblick Bei der Beurteilung der Qualität von Regenwasserabflüssen, der Entsorgungswege und Boden- und Gewässerbelastung sollte dem zunehmenden Einsatz von Bioziden und anderen Additiven in Baumaterialien als mögliche Belastungsquelle verstärkt Beachtung geschenkt werden. Ein ganzheitliches Management von Baumaterialien und der Regenwasserbewirtschaftung ist gefordert. Die noch laufenden Labor- und Feldstudien werden weitere Ergebnisse zur Auswaschung von Bioziden und Additiven aus Fassadenfarben und -putzen sowie Dachmaterialien liefern.

Schlüsselwörter Auswaschung · Baumaterialien · Biozide · Carbendazim · Cybutryn · DCOIT · Diuron · Fassaden · IPBC · Irgarol $^{\circledR} 1051 \cdot$ Modellierung $\cdot$ OIT $\cdot$ Ökotoxikologische Effekte $\cdot$ Risikobewertung $\cdot$ Terbutryn $\cdot$ Zinkpyrithion

\section{Biocides in building facades - ecotoxicological effects, leaching and environmental risk assessment for surface waters}

Abstract Background, aim and scope Sources of organic micropollutants occuring in surface waters are often unknown. Regarding environmental risk assessments for surface waters, construction materials have as till now, not been given much consideration, although biocides used as preservatives are known to reach urban storm water runoff. The study focused on biocides for facades coatings and aimed (1) to determine ecotoxicological effect values, (2) to quantify the leaching behaviour and (3) to assess the environmental risk for surface waters using a dynamic transport model.

Materials and methods Eight biocides used in resin based facade coatings were investigated. Some biocides are substances known as pesticides for agricultural purposes like diuron, carbendazim and terbutryn. Ecotoxicological effect values for aquatic organisms were determined for every biocide. Leaching of four biocides from a render under UV-irradiation has been investigated in the laboratory including the influence of varying temperatures. Using 80 irrigation intervals over 28 days, facade runoff was sampled and followed by biocide chemical analysis. The total losses were calculated based on the concentration patterns. These data were used for modelling the transport of cybutryn from facades to surface waters. Biocide specific effect values and leaching characteristics have been taken into consideration.

Results Acute and chronic effect values as well as predicted no effect concentrations for the investigated biocides indicate their high potential to affect aquatic organisms. The leaching of four biocides (diuron, terbutryn, cybutryn, carbendazim) from the facade render under the experimental conditions delivers high concentrations in the beginning followed by an exponential decrease. Rising temperature increased the concentration of biocides in the runoff. The total losses were between $7 \%$ and $29 \%$ depending on the substances. More than half of the losses occur in the runoff within the first $15 \mathrm{~min}$ of runoff from a 60 min irrigation cycle. The modelling result for cybutryn underlines its high environmental risk for small surface waters.

Discussion The leaching of the biocides, their potential ecotoxic effects and persistence show clearly that the environmental risk for surface waters and soils seems to be high for certain biocides; whereas for others the risk seems to be significantly lower. With respect water quality criteria, polluted facades runoff has to be diluted before runoff can enter the discharge. Diuron and carbendazim are however also used as pesticides and preservatives for other materials and cybutryn is also used as an antifouling agent. All pathways have to be evaluated in order to identify relevant sources and to act more efficiently with respect to water and soil protection.

Conclusions Concentrations with high environmental risk are expected at new facades, especially at facades with thermal insulation. With the given low predicted no effect concentrations in a range of a few $\mathrm{ng} / \mathrm{L}$ and large amounts of biocides applied in paints and renders, the environmental 
risk for common biocides used in facade coatings has to be investigated in laboratory and field scale. It seems plausible that source control measures as the most efficient and sustainable precautionary principle need to be evaluated.

Recommendations and perspectives Biocides and additives applied in construction materials have to be taken into consideration as relevant sources when evaluating the quality of storm water runoff, discharge into urban areas and the impact to soil and surface waters. A sustainable construction material management and storm water management are required. It is expected that ongoing laboratory and field studies with exterior paints, renders and flat sheets for waterproofing containing biocides and additives will give further insight into their environmental impact.

Keywords Biocides $\cdot$ Carbendazim $\cdot$ Construction products · Cybutryn · DCOIT - Diuron · Ecotoxicological effects $\cdot$ Environmental risk assessment $\cdot$ Facades $\cdot$ IPBC · Irgarol ${ }^{\circledR} 1051 \cdot$ Leaching $\cdot$ Modelling $\cdot$ OIT $\cdot$ Terbutryn $\cdot$ Zinc pyrithione

\section{Hintergrund}

Biozidprodukte werden in Baumaterialien als Materialschutzmittel gegen Algen, Pilze und Mikroorganismen eingesetzt. Für die Schweiz wird angenommen, dass von den jährlich verbrauchten $7.400 \mathrm{t}$ Bioziden rund $25 \%$ als Schutzmittel für Baumaterialien verwendet werden (Bürgi et al. 2008). Die Anwendung ist in der Schweiz nach der Biozidprodukteverordnung (Schweizerischer Bundesrat 2005) reguliert, welche in Anlehnung an die Europäische Biozidproduktenrichtlinie formuliert ist (Europäische Union 1998). Die Schutzmittel in Baumaterialien, Hauptgruppe 2 der Biozidproduktenrichtlinie, lassen sich nach ihrer Anwendung in Topf-Konservierungsmittel (Produktart 6), Beschichtungsschutzmittel (Produktart 7), Schutzmittel für Fasern, Leder, Gummi und polymerisierte Materialien (Produktart 9), Holzschutzmittel (Produktart 8) und Schutzmittel für Mauerwerk (Produktart 10) gliedern. Zu den Baumaterialien, die mit diesen Schutzmitteln behandelt werden können, zählen beispielsweise Beton, Eternit, Holz, Dichtungsmassen, Kunststoffdichtungsbahnen sowie Farben und Putze für Fassaden. Die Ausrüstung von kunstharzgebundenen Farben und Putzen mit Bioziden entspricht bei wärmegedämmten Fassadensystemen sogar dem Stand der Technik. In Farben und Putzen werden pro Kilogramm Material zwischen 100 bis 2000 mg Wirkstoff eingesetzt. Basierend auf den durchschnittlichen Anwendungsmengen wurde für die Schweiz allein dieser Biozidverbrauch auf jährlich 60 bis $300 \mathrm{t}$ geschätzt (Bürgi et al. 2008). Es ist anzunehmen, dass die Anwendung von Bioziden in Fassaden weiter zunimmt (Burkhardt et al. 2006).
Diskutiert wird auch der Biozideinsatz in Dachfarben (Menge 2005).

Die meisten Schutzmittel, die unter der Hauptgruppe 2 reguliert sind, enthalten nur wenige verschiedene biozide Wirkstoffe. Dies ist auch darauf zurückzuführen, dass die gleichen Wirkstoffe in unterschiedlichen Produktarten eingesetzt werden. Von 22 als prioritär eingestuften Bioziden in der Schweiz - die Priorisierung erfolgte nach Bürgi et al. (2008) - kommen sogar rund $90 \%$ in Baumaterialien vor. Dazu zählen beispielsweise Diuron, das auch als prioritäre Substanz in der EU-Wasserrahmenrichtlinie und als gefährlicher Bauinhaltsstoff (European Committee for Standardization 2006) gelistet ist, und Terbutryn, das seit 2003 in der EU nicht mehr als Pestizid zugelassen ist.

Biozid ausgerüstete Baustoffe sind aus Sicht des Umwelt- bzw. Gewässerschutzes besonders dann zu beachten, wenn die Materialien der natürlichen Witterung ausgesetzt sowie ein wesentlicher Austrag in die Umwelt und ökotoxikologisches Risiko für Organismen zu erwarten sind. Mit einer in Überarbeitung befindlichen Bauproduktenrichtlinie (Europäische Union 1988) soll dem potentiellen Belastungsrisiko begegnet werden. Zukünftig soll die Auswaschung von Problemstoffen (,hazardous substances') experimentell geprüft und bewertet werden (CEN/TC351, ER3). Wenn Biozide aus exponierten Materialien ausgewaschen werden, so ist mit deren Vorkommen vor allem im Regenabwasser von urbanen Räumen zu rechnen. Als relevante Wege in den Boden und die Gewässer gelten (1) die dezentrale Regenwasserversickerung an Gebäuden, (2) die Regenwasserableitung via Trennkanalisationen in Fliessgewässer und (3) die Regenwasserentlastung von Kläranlagen bei Regenwetter (Burkhardt et al. 2007). Deshalb ist wenig überraschend, dass als prioritär eingestufte Biozide im Klärschlamm (Kupper et al. 2006), in Kläranlagenabläufen (Gerecke et al. 2001) und in Gewässern (Balsiger et al. 2007) als Mikroverunreinigungen nachgewiesen wurden. Dazu zählen beispielsweise Diuron, Carbendazim, Terbutryn und Cybutryn (Synonym: Irgarol ${ }^{\circledR} 1051$ ).

Eine umfassende Messkampagne in zwei Zürcher Fließgewässern zeigte jüngst eindrucksvoll, dass sich das Vorkommen von einigen Bioziden deutlich vom charakteristischen saisonalen Auftreten der Pestizide unterscheidet, welche in der Landwirtschaft eingesetzt werden (Steinmann et al. 2008). Das ganzjährige Auftreten von beispielsweise Cybutryn in einem Bach wurde daher als Hinweis auf urbane Quellen interpretiert, aus denen unabhängig von der Jahreszeit der Wirkstoff freigesetzt wird. Als potentielle Quelle für Cybutryn konnte die Anwendung als Antifouling (Konstantinou und Albanis 2004) a priori ausgeschlossen werden. Quednow und Püttmann et al. (2007) diskutierten in einem vergleichbaren Zusammenhang, ob das verbreitete Auftreten von Terbutryn in Fließgewässern eher auf den Austrag von landwirtschaftlichen Nutzflächen oder die 
Desorption vom Gewässersediment zurückgeführt werden kann. Dabei wurde kaum berücksichtigt, dass Terbutryn ein wichtiges Biozid im Fassadenschutz von Gebäuden darstellt.

In die Beurteilung der ökologischen Nachhaltigkeit von Baumaterialien sollte ebenfalls das ökotoxische Risiko der eingesetzten Wirkstoffe auf aquatische oder terrestrische Organismen einfließen. Für einige biozide Wirkstoffe sind ökotoxische Wirkungen bekannt (z. B. Arrhenius et al. 2006; Dahl und Blanck 1996; Schmidt et al. 2007), für viele fehlen aber ökotoxische Schwellenwerte, die beispielsweise als Qualitätsziele im Gewässer angewendet werden können. Im Rahmen einer umfassenden Literaturstudie lassen sich jedoch beachtliche Datengrundlagen erarbeiten und Abschätzungen zur Wirkung herleiten. Schmidt et al. (2007) zeigen darüber hinaus, dass mit einer kombinierten Expositions- und Effektuntersuchung das Belastungsrisiko für Boden und Gewässer aussagekräftig abgeleitet werden kann.

Die punktuellen Nachweise in Gewässern unterstreichen, dass die Belastungen diffus und mit hoher zeitlicher und räumlicher Variabilität auftreten. Daher lässt sich der Stofffluss in urbanen Entwässerungssystemen von der Quelle bis ins Gewässer quantitativ nur mit erheblichem experimentellen Aufwand erfassen und eine Bilanzierung im urbanen Regenwasserabfluss muss im Vergleich zur Betrachtung des Pestizidaustrags aus der Landwirtschaft zusätzliche Aspekte berücksichtigen (Goonetilleke et al. 2005). Zudem lassen sich die Quellen für weitere Stoffe, welche in urbanen Regenabflüssen vorkommen können, vielfach nur mit großer Unsicherheit ermitteln (Eriksson et al. 2007). Aus einer Messkampagne in der Schweiz wurde dennoch gefolgert, dass der urbane Eintrag in Gewässer dem Eintrag aus der Landwirtschaft durchaus ebenbürtig sein dürfte (Gerecke et al. 2001).

\section{Ziel}

Im Rahmen der Arbeit sollte die (1) Ökotoxizität von acht Bioziden ermittelt, (2) deren Auswaschung aus kunstharzgebundenen Putzsystemen unter vergleichbaren experimentellen Randbedingungen quantifiziert und (3) mittels einer Stofftransportmodellierung das Belastungsrisiko für Gewässer grob abgeschätzt werden. Zunächst sollten für alle Wirkstoffe die vorhergesagten Konzentrationen ohne Effekt (PNEC) und der Toxizitätsschwellenwert HC05 mit etablierten Methoden der Ökotoxikologie evaluiert werden. Anschließend sollten für vier Biozide Massenbilanzen im Fassadenablaufwasser exemplarisch eine Abschätzung zum Eintrag in das abfließende Regenwasser ermöglichen und mittels Stofftransportmodellierung auf ein Einzugsgebiet übertragen werden.

\section{Material und Methoden}

\subsection{Substanzauswahl}

Zum fungiziden und algiziden Schutz von kunstharzgebundenen Fassadenfarben und -putzen steht eine relativ kleine Auswahl von Bioziden mit unterschiedlichen Wirkspektren und -mechanismen zur Verfügung (Tabelle 1) (Wunder 2001). Damit ein umfassender Fassadenschutz gewährleistet ist, werden bis zu fünf Biozide als Mischungen kombiniert, bestehend aus Wirkstoffen unterschiedlicher Wasserlöslichkeit und Wirkungsspektren. Heute werden vor allem Terbutryn, Zinkpyrithion, Octyl-Isothiazolinon (OIT), Carbendazim, Jodpropinylbutylcarbamat (IPBC), Diuron und Cybutryn eingesetzt, seltener dagegen Dichloro-Octyl-Isothiazolinon (DCOIT). Diese Wirkstoffe sind auch aus anderen Anwendungen bekannt. So werden Diuron und Carbendazim auch als Pestizide in der Landwirtschaft eingesetzt, Terbutryn dagegen ist als Pestizid in der EU bereits seit 2003 nicht mehr zugelassen (seit 2008 auch in der Schweiz). Die Isothiazolinone, IPBC und Zinkpyrithion sind auch durch Anwendungen in Kosmetikprodukten bekannt. Cybutryn und Terbutryn gehören zur Wirkstoffgruppe der Triazine, zu der auch Atrazin zählt. Wie auch Diuron hemmen diese Wirkstoffe reversibel die Photosynthese. Die Isothiazolinone, IPBC und Zinkpyrithion zeichnen sich durch eine breite, irreversible Wirksamkeit gegen Algen, Pilze und Mikroorganismen aus.

\subsection{Vorgehen zur Bewertung von ökotoxikologischen Effekten}

Die ökotoxikologische Beurteilung der Gewässerqualität basiert auf effektbezogenen Grenzkonzentrationen. Um für die acht betrachteten Biozide (Tabelle 1) Grenzwerte abzuleiten, wurde zunächst eine umfassende Literaturrecherche im Web of Science und der Datenbank des ,Pesticide Action Network' (http://pesticideinfo.org) durchgeführt. Die in den Publikationen aufgeführten Toxizitätsdaten wurden in eine Datenbank überführt. Verwendet wurden ausschließlich Daten für wasserlebende Organismen. Auf Grundlage dieser Daten wurde jeweils die Konzentration, die keine schädigende Wirkung erwarten lässt (Predicted No Effect Concentration, PNEC), abgeleitet. Die Ableitung erfolgte auf drei verschiedenen Wegen: (1) Sind bereits verlässliche Wasserqualitätsziele publiziert, wurden diese übernommen; (2) Lagen in Anlehnung an das ,Technical Guidance Document on Risk Assessment ${ }^{\star}$ TGD (European Chemicals Bureau 2003) wenigstens 10 NOEC-Daten (höchste getestete Konzentration ohne Effekt) für 8 unterschiedliche taxonomische Gruppen vor, wurden Spezies-Sensitivitätsverteilungen (SSDs) berechnet; (3) War die Datenlage für die Berechnung einer SSD nicht ausreichend, wurden die PNECs basierend auf 
Tabelle 1 Stoffeigenschaften von Bioziden, die in Fassadenbeschichtungen sowie anderen Produktarten eingesetzt werden

\begin{tabular}{|c|c|c|c|c|c|c|}
\hline & CAS-Nr. & Strukturformel & $\begin{array}{c}\text { Wasserlöslichkeit } \\
(\mathrm{mg} / \mathrm{L})^{\mathrm{a}}\end{array}$ & Wirkung & Produktarten ${ }^{\mathrm{b}}$ & Pestizid \\
\hline Diuron & $330-54-1$ & & 42 & Algizid & $6,7,10$ & $\mathrm{Ja}$ \\
\hline $\begin{array}{l}\text { Cybutryn } \\
\left.\text { (Irgarol }^{\circledR} 1051\right)\end{array}$ & 28159-98-0 & & 7 & Algizid & $7,9,10,21$ & - \\
\hline Terbutryn & $886-50-0$ & & 22 & Algizid & $7,9,10$ & $\begin{array}{l}\text { seit Juli } 2003 \\
\text { in der EU } \\
\text { nicht mehr } \\
\text { zugelassen }\end{array}$ \\
\hline Carbendazim & $10605-21-7$ & & 8 & Fungizid & $\begin{array}{l}6,7,9,10,11 \\
12,13\end{array}$ & $\mathrm{Ja}$ \\
\hline IPBC & $55406-53-6$ & & 168 & Fungizid & $\begin{array}{l}6,7,8,9,10,11 \\
13,18\end{array}$ & - \\
\hline OIT & $26530-20-1$ & & 500 & $\begin{array}{l}\text { Fungizid, } \\
\text { Algizid }\end{array}$ & $\begin{array}{l}6,7,89,10,11, \\
12,13\end{array}$ & - \\
\hline DCOIT & $64359-81-5$ & & 14 & $\begin{array}{l}\text { Fungizid, } \\
\text { Algizid }\end{array}$ & $\begin{array}{l}6,7,9,10,11 \\
12,21\end{array}$ & - \\
\hline Zinkpyrithion & $13463-41-7$ & & 8 & $\begin{array}{l}\text { Fungizid, } \\
\text { Algizid }\end{array}$ & $6,7,9,10,13,21$ & - \\
\hline
\end{tabular}

${ }^{\text {a }}$ Die Wasserlöslichkeit ist aus Paulus et al. (2004) entnommen

${ }^{\mathrm{b}}$ Produktarten der Biozidproduktenverordnung (Europäische Union 1998)

dem tiefsten NOEC (gegebenenfalls auf dem tiefsten EC50) unter Anwendung der in der TGD beschriebenen Sicherheitsfaktoren abgeleitet (Sicherheitsfaktoren-Methode).

Die Ableitung der SSDs basiert auf dem Vorgehen, welches vom European Chemicals Bureau (2003) definiert wurde und beispielsweise von Chèvre et al. (2006) angewendet wurde. Bei der Berechnung von SSDs ist entscheidend, dass alle verwendeten taxonomischen Gruppen prinzipiell gleich empfindlich gegenüber dem untersuchten Stoff sind. Wenn dies nicht der Fall ist, treten häufig bi-phasische Verteilun- gen auf, anhand derer keine PNECs abgeleitet werden sollten (Posthuma et al. 2002). Meistens war die Datenlage für EC50-Daten besser als für NOECs, so dass zunächst anhand einer EC50-SSD die bi-phasische Verteilung der Empfindlichkeit geprüft wurde. In Fällen, in denen sich einzelne taxonomische Gruppen als deutlich empfindlicher erwiesen als andere, wurde die SSD daher nur für die empfindlichere Gruppe erstellt. Die verwendeten Toxizitätsdaten und jeweilige Ableitung der PNECs wurden im Online Appendix (Electronic Supplementary Material) zusammengestellt. 
Aus der jeweiligen SSD wurde der HC05-Wert abgeleitet, eine Konzentration bei der für $5 \%$ der betrachteten Spezies der gewählte Endpunkt (NOEC oder EC50) überschritten wurde bzw. 95\% vor dem Auftreten dieser Endpunkte geschützt werden. Der $\mathrm{HC} 05_{\mathrm{EC} 50}$ wird vielfach als ein akuter Toxizitätsschwellenwert und der $\mathrm{HC} 05_{\mathrm{NOEC}}$ als chronischer Wert definiert. In der Schweiz wurde in diesem Zusammenhang von Chèvre et al. (2006) vorgeschlagen, dass in Gewässern das akute Qualitätskriterium zu keinem Zeitpunkt und das chronische nicht in kleineren Abständen als zwei Wochen überschritten werden darf. Entgegen dem Vorgehen von Chèvre et al. (2006) wurde für die hier vorgestellte Ableitung der PNECs nicht das 95\% Vertrauensintervall des HC05 berechnet, sondern in Anlehnung an die TGD (European Chemicals Bureau 2003) die ermittelten $\mathrm{HC} 05_{\mathrm{NOEC}}$ durch einen Sicherheitsfaktor von 5 geteilt.

$\mathrm{Zu}$ Vergleichszwecken wurde für DCOIT auch eine SSD mit weniger Datenpunkten berechnet, der abgeleitete PNEC aber ergänzend durch die Sicherheitsfaktoren-Methode abgestützt. Für Cybutryn wurden die Toxizitätsschwellenwerte darüber hinaus nach der Extrapolationsmethode von Chèvre et al. (2006) berechnet. Hierdurch sollte für wirkungsgleiche Stoffe geprüft werden, ob die Extrapolationsmethode zu zuverlässigen Ergebnissen führt. Details zur Extrapolationsmethode sind in Chèvre et al. (2006) beschrieben.

\subsection{Laborversuche zur Auswaschung}

In einem Laborversuch wurde die Auswaschung von Bioziden unter Berücksichtigung der UV-Bestrahlung und Beregnungsmenge quantifiziert und dabei die Temperatur variiert. Die Nutzung einer Bewitterungskammer ermöglichte die Untersuchung einer vertikal installierten Fassadenpanele im halbtechnischen Maßstab mit $2.0 \mathrm{~m}$ Höhe und $0.75 \mathrm{~m}$ Breite. Für den Versuch wurde ein marktüblicher, aber biozidfreier Deckputz mit den acht Bioziden dotiert. Die Wirkstoffsmenge im Putz lag bei $500 \mathrm{mg} / \mathrm{kg}$ je Biozid und bezogen auf die behandelte Fläche bei $1500 \mathrm{mg} / \mathrm{m}^{2}$ je Biozid. Die einheitliche Konzentration im Putz erlaubt eine unmittelbare Interpretation des Vorkommens im Fassadenablauf. In marktüblichen Produkten liegen die Konzentrationen zwischen $100 \mathrm{mg} / \mathrm{kg}$ und $2000 \mathrm{mg} / \mathrm{kg}$ je Wirkstoff. Der ausgerüstete Deckputz wurde von einem Malereibetrieb praxisgerecht auf einer Wärmedämmplatte aus extrudiertem Polystyrol (EPS) aufgetragen. Die Dämmplatte war im Vorwege mit einem Unterputz vorbereitet worden. Praxisübliche Standzeiten von 2 Wochen für den Unterputz und 5 Tagen für den Deckputz wurden beachtet.

Die gesamte Bewitterungsdauer setzt sich aus vier Zyklen mit jeweils 20 Beregnungsintervallen in fünf Tagen und nachfolgenden zwei Ruhetagen zusammen. Die Auswaschung aus den Fassadenpanelen wurde demnach über
80 Beregnungsintervalle in 28 Tagen untersucht. Während jeder Beregnung wurde die Fassadenoberfläche mit jeweils $85 \mathrm{~L} / \mathrm{m}^{2}$ Wasser in 1 Stunde gleichmäßig benetzt. Die Versuchsbedingungen wurden in Anlehnung an die Europäische Richtlinie zur Prüfung von Fassadenbeschichtungen ETAG 004 gewählt (EOTA 2000). Die Beregnungsmenge von $6800 \mathrm{~mm}$ entspricht annähernd dem sechsfachen Jahresniederschlag im Kanton Zürich. Bereits damit wird deutlich, dass eine stark beschleunigte Auswaschung realisiert wurde. Im ersten und dritten Zyklus lag auf der Fassadenoberfläche die Temperatur bei $+50^{\circ} \mathrm{C}$ und im zweiten und vierten Zyklus wurde die Temperatur auf $+60^{\circ} \mathrm{C}$ erhöht. Diese Temperaturen können an realen Gebäudefassaden bei direkter Sonneneinstrahlung erreicht werden. Das Fassadenwasser wurde in definierten Zeitabständen zeitproportional und automatisiert beprobt. Die Biozide wurden mittels Flüssigchromatographie-Tandem-Massenspektrometrie (LC-MS/MS) analysiert. Zur Qualitätssicherung wurden isotopenmarkierte interne Standards verwendet. Vorgestellt werden erste Ergebnisse von vier Bioziden (Diuron, Terbutryn, Cybutryn und Carbendazim), welche sich durch eine vergleichsweise hohe Persistenz und Ökotoxizität auszeichnen.

\subsection{Belastungsabschätzung für Gewässer mittels Stofftransportmodellierung}

Ein Niederschlags-Abfluss-Modell wurde für eine Überbauung nahe Zürich erstellt und kalibriert. Nachfolgend wurde der Stofftransport ins Gewässer via Regenwasserabfluss für ein urbanes Einzugsgebiet von 11 ha Fläche dynamisch modelliert. Als Quellenfunktion für das Biozid Cybutryn fungierten sieben westexponierte Fassadenbeschichtungen mit rund $3500 \mathrm{~m}^{2}$ Gesamtfläche. Bei der Auswaschung werden nur diese Fassadenflächen, der Anteil des Fassadenabflusses am Jahresniederschlag und die Auswaschrate berücksichtigt. Im vorgestellten Szenario wurde angenommen, dass alle sieben Fassadenflächen mit Cybutryn ausgerüstet sind $\left(690 \mathrm{mg} / \mathrm{m}^{2}\right)$ und von der eingesetzten Wirkstoffmenge über $1 \mathrm{Jahr} 12 \%$ und nach 5 Jahren $20 \%$ exponentiell abnehmend ausgewaschen werden. Das zugrundeliegende Entwässerungssystem ist so definiert, dass der belastete Fassadenabfluss über Drainagen in den Regenwasserkanal der Überbauung und des anschließenden Einzugsgebiets fließt, der direkt in den Vorfluter einleitet. Das fiktive nicht verschmutzte Gewässer weist einen Abfluss von $500 \mathrm{~m}^{3} / \mathrm{h}$ auf. Die gezeigte Niederschlagsverteilung entspricht realen Daten für das Jahr 2003. Vorgestellt wird die Simulationsrechnung zum Vorkommen des Biozids im Gewässer unter Berücksichtigung des akuten Wasserqualitätsziels für den Zeitraum Januar bis Mai 2003. Details zur Modellierung sind in Walser et al. (2008) präsentiert. 


\section{Ergebnisse und Diskussion}

\section{1 Ökotoxikologische Effekte von Bioziden}

Wie für andere s-Triazine beruht der ökotoxische Effekt von Cybutryn vor allem auf der spezifischen Hemmung des Photosystems II. Da nur Cyanobakterien (Blaualgen), Algen und Pflanzen Photosynthese betreiben, Tiere und Bakterien hingegen nicht, ist zu erwarten, dass Algen und Wasserpflanzen besonders empfindlich auf Cybutryn reagieren. In Abbildung 1 (links) wird deutlich, dass die EC50-SSD für alle Spezies bi-phasisch ist (Abb. 1, links: Kästchen). Sie setzt sich einerseits aus der Empfindlichkeitsverteilung für photosyntheseaktive Organismen (Dreieck: Pflanzen und Blaualgen), und andererseits für Tiere und Bakterien (Abb. 1, links: Kreis) zusammen. Pflanzen und Blaualgen reagieren auf Cybutryn rund drei Größenordnungen empfindlicher als Tiere und Bakterien. Daher wurden für die Ableitung des Toxizitätsschwellenwertes nur die Daten für Pflanzen und Blaualgen berücksichtigt. Zwischen marinen und limnischen Algen besteht kein deutlicher Unterschied, so dass beide Datensätze zusammen betrachtet wurden. An der Gesamtübersicht wird deutlich (Abb. 1, links), dass bei artspezifischen Wirkmechanismen eine Differenzierung nach bestimmten Spezies sehr wichtig ist. In diesen Fällen können SSDs auch auf weniger als den geforderten 8 taxonomischen Gruppen basieren (European Chemicals Bureau 2003). Nur mit den sensitivsten taxonomischen Gruppen können aussagekräftige Toxizitätsschwellenwerte abgeleitet werden.

Der $\mathrm{HCO5}_{\mathrm{EC} 50}$ für Cybutryn liegt für den besten Fit - die SSD, die direkt aus den ökotoxikologischen Daten berech- net und mit einem log-logistischen Modell gefittet wurde - bei $110 \mathrm{ng} / \mathrm{L}$ und für den $\mathrm{HC} 05_{\mathrm{NOEC}}$ bei $5 \mathrm{ng} / \mathrm{L}$ (Abb. 1, rechts). Ein Vergleich mit NOECs für marine Algengemeinschaften - 16 bis $530 \mathrm{ng} / \mathrm{L}$ (Arrhenius et al. 2006; Dahl et al. 1996) - zeigt, dass sich der $\mathrm{HC} 05_{\text {NOEC }}$ und die NOECs der empfindlicheren Gemeinschaften in der gleichen Größenordnung bewegen. Wird in Anlehnung an die TGD (European Chemicals Bureau 2003) auf den $\mathrm{HC} 05_{\mathrm{NOEC}}$ ein Sicherheitsfaktor von 5 gelegt, ergibt sich ein abgeleiteter PNEC von $1 \mathrm{ng} / \mathrm{L}$. Angesichts der Tatsache, dass die analytischen Nachweisgrenzen von LC-MS/MS im Bereich des PNEC liegen, dürfte sich mit jedem analytischen Nachweis in der Umwelt bereits eine Wirkung auf Algen verbinden. In Tabelle 2 sind die ermittelten ökotoxischen Effektkennzahlen zusammengestellt.

Nicht für jeden Stoff sind genügend NOEC-Werte für die Ableitung eines $\mathrm{HC} 05_{\mathrm{NOEC}}$ verfügbar. Da aber angenommen werden kann, dass die relative Empfindlichkeit der Arten gegenüber zwei Stoffen mit demselben Wirkmechanismus identisch ist, haben Chèvre et al. (2006) eine Methode entwickelt, mit der ein $\mathrm{HC} 05_{\mathrm{NOEC}}$ vom $\mathrm{HC} 05_{\mathrm{NOEC}}$ einer wirkungsgleichen Referenzsubstanz abgeleitet werden kann. Mit der SSD für Cybutryn, die den Datenanforderungen der TGD vollumfänglich entspricht, konnte nun die Extrapolationsmethode von Chèvre et al. (2006) unabhängig geprüft werden. Zur Ableitung dieser zweiten SSD von Cybutryn wurde Atrazin - mit dem Datensatz aus Chèvre et al. (2006) als Referenzsubstanz gewählt. Wie in Abbildung 1 gezeigt wird, ist die Vorhersage der NOEC-SSD für Cybutryn auBerordentlich gut. Da sich die Steigung der vorhergesagten SSD von der ersten SSD geringfügig unterscheidet, beträgt
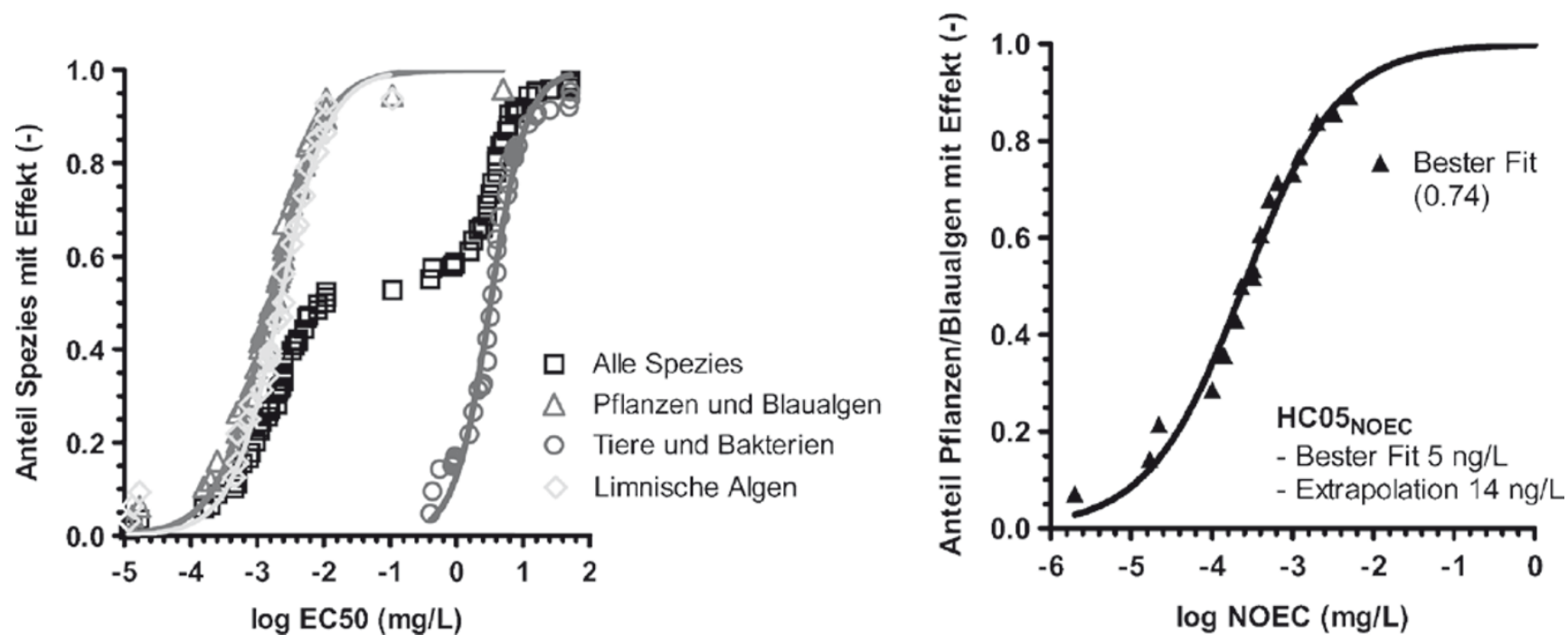

Abb. 1 Spezies-Sensitivitätsverteilungen (SSDs) für EC50-Daten von Cybutryn für alle Spezies (links) und NOEC-SSDs für die empfindlichsten Arten, Pflanzen und Blaualgen, mit zwei Methoden im Vergleich (rechts) - einerseits anhand der Effektdaten für Algen (bester Fit) und andererseits basierend auf der Extrapolationsmethode nach Chèvre et al. (2006) 
Tabelle 2 Konzentrationen HC05 für die empfindlichsten taxonomischen Gruppen sowie PNECs, die entweder von den HC05 Werten oder dem tiefsten EC50/NOEC abgeleitet wurden

\begin{tabular}{lcccc}
\hline Wirkstoff & $\begin{array}{c}\mathbf{H C O 5}_{\text {EC50 }} \\
(\mathbf{n g} / \mathbf{L})\end{array}$ & $\begin{array}{c}\mathbf{H C 0 5}_{\text {NOEC }} \\
(\mathbf{n g} / \mathbf{L})\end{array}$ & $\begin{array}{c}\text { Ableitung } \\
(\mathbf{m i n} .)\end{array}$ & $\begin{array}{c}\text { PNEC } \\
(\mathbf{n g} / \mathbf{L})\end{array}$ \\
\hline Cybutryn & 110 & 5 & - & 1 \\
Diuron & & $100^{\mathrm{a}}$ & - & 20 \\
Terbutryn & & $170^{\mathrm{a}}$ & - & 34 \\
DCOIT & 110 & $37^{\mathrm{b}}$ & $\mathrm{NOEC}$ & $8^{\mathrm{b}} / 8$ \\
Zinkpyrithion & & $\mathrm{c}$ & $\mathrm{EC50}$ & 2,6 \\
IPBC & & $\mathrm{c}$ & $\mathrm{EC50}$ & 26 \\
Carbendazim & & $\mathrm{c}$ & $\mathrm{NOEC}$ & 34 \\
OIT & $\mathrm{c}$ & $\mathrm{EC50}$ & 13 \\
\hline
\end{tabular}

a aus: Chèvre et al. (2006)

b erfüllt nur teilweise die Kriterien vom Technical Guidance Document on Risk Assessment (TGD) (European Chemicals Bureau 2003)

c Anzahl NOEC-Daten nicht ausreichend für SSD

der extrapolierte $\mathrm{HC} 05_{\mathrm{NOEC}} 14 \mathrm{ng} / \mathrm{L}$ und der direkt bestimmte $\mathrm{HC} 05_{\mathrm{NOEC}} 5 \mathrm{ng} / \mathrm{L}$. Für die regulatorische Anwendung ist dieser Unterschied beinahe vernachlässigbar. Der Vergleich liefert damit den ersten empirischen Beweis für die Gültigkeit der Extrapolationsmethode für wirkungsgleiche Stoffe und bestätigt deren Potential als wertvolle Methode in der ökotoxikologischen Risikobeurteilung.

Für DCOIT wurden 25 EC50 Daten für 13 Spezies aus 8 taxonomischen Gruppen (Algen, Fische, Bakterien, Krebstiere, Mollusken, Echinodermaten, Ascidien, Makrophyten) recherchiert. Davon waren 7 Spezies marin und 6 limnisch. Abbildung 2 (links) zeigt die SSD für die EC50 Werte. Die breite Datenbasis ist recht homogen über die SSD verteilt. Es ist keine taxonomische Gruppe erkennbar, die besonders empfindlich gegenüber DCOIT ist. Der $\mathrm{HC} 05_{\mathrm{EC} 50}$ beträgt $110 \mathrm{ng} / \mathrm{L}$ und wurde wiederum mit einem log-logistischen Modell abgeleitet. Zur chronischen Toxizität sind nur NOEC-Daten für 5 Spezies aus 3 taxonomi- schen Gruppen (Algen, Krebstiere, Cyanobakterien) gefunden worden (Abb. 2 rechts). Die Anforderungen der TGD für die Ableitung eines PNEC mit der SSD Methode waren damit nicht voll erfüllt. Der abgeleitete $\mathrm{HC} 05_{\mathrm{NOEC}}$ liegt bei $37 \mathrm{ng} / \mathrm{L}$. Mit dem Sicherheitsfaktor 5 ergibt sich ein PNEC von $8 \mathrm{ng} / \mathrm{L}$. Wenn dazu alternativ auf den niedrigsten gefundenen NOEC entsprechend der TGD der Sicherheitsfaktor von 50 anwendet wird, wird exakt der gleiche PNEC erhalten. Damit ergeben zwei Methoden - die Erstellung einer SSD mit kleiner Datenbasis und die Berücksichtigung eines Sicherheitsfaktors - den gleichen PNEC.

Die Datenbasis für die Wirkstoffe Carbendazim, Zinkpyrithion, IPBC und OIT war so ungenügend, dass keine der SSDs den Anforderungen der TGD entsprochen hätte. Die PNECs wurden daher unter Berücksichtigung der geforderten Sicherheitsfaktoren (European Chemicals Bureau 2003) von den tiefsten EC50- oder NOEC-Daten abgeleitet. Die $\mathrm{HC} 05_{\mathrm{NOEC}}$ für Diuron und Terbutryn wurden von Chèvre et al. (2006) übernommen und der PNEC mit einem Sicherheitsfaktor von 5 berechnet (s. Tabelle 2). Die wirkungsgleichen Substanzen Diuron, Terbutryn und Cybutryn weisen im Übrigen durchwegs deutlich tiefere Qualitätskriterien auf als das in der EU seit 2004 und in der Schweiz seit 2007 nicht mehr zugelassene Herbizid Atrazin. Für Cybutryn liegt der Wert sogar rund 100mal niedriger als für Atrazin.

\subsection{Auswaschungsverhalten von Bioziden aus Fassadenputz}

Biozide sind nur wirksam, wenn sie aus der Fassadenmatrix in die wässerige Phase gelangen und über diesen Weg die Zielorganismen erreichen können. Biozide können an die Oberfläche von Fassaden diffundieren, wenn ausreichend Feuchte in der Beschichtung vorliegt. Die Feuchte kann einerseits mit dem Niederschlag in die Beschichtung gelangen, andererseits während regenfreier Zeiten auch über Kondens- und Tauwasser. Jedes abflusswirksame Schlagre-
Abb. 2 Spezies Sensitivitätsverteilung (SSD) für DCOIT basierend auf log EC50-Werten für 13 Spezies aus 8 taxonomischen Gruppen (links; $\mathrm{n}=25$ ) und der SSD basierend auf logarithmierten NOEC-Werten für 5 Spezies aus 3 taxonomischen Gruppen (rechts; $\mathrm{n}=5$ )
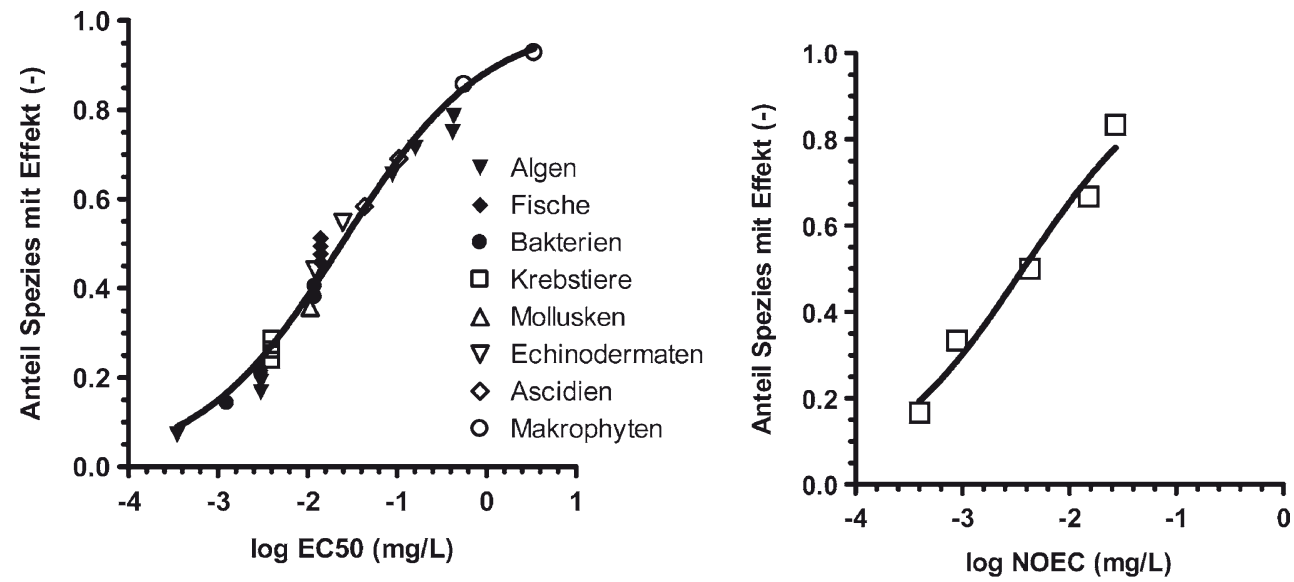
genereignis transportiert die Biozide an der Fassade abwärts. Die Anzahl an Fassadenabflussereignissen wird auch durch die Exposition beeinflusst, so dass an stärker bewitterten Oberflächen die Auswaschung schneller erfolgen wird.

Im Laborversuch wurde eine dichte Abfolge von 80 Schlagregenintervallen in 28 Tagen untersucht. Seit vielen Jahren wird eine derartige Intensivbewitterung für die hygrothermische Prüfung von Fassadensystemen angewandt. Unter diesen Versuchsbedingungen nahmen im Fassadenabfluss die Konzentrationen von den vier Bioziden Diuron, Cybutryn, Terbutryn und Carbendazim über beinahe zwei Größenordnungen exponentiell ab (Abb. 3). Die Konzentrationen lagen im ersten Liter des ersten Fassadenabflusses zwischen $7000 \mu \mathrm{g} / \mathrm{L}$ für Diuron und $700 \mu \mathrm{g} / \mathrm{L}$ für Carbendazim. Im ersten Liter des achtzigsten Abflussereignisses wurden nur noch $80 \mu \mathrm{g} / \mathrm{L}$ Diuron und $40 \mu \mathrm{g} / \mathrm{L}$ Carbendazim nachgewiesen.

Die hohen Konzentrationen in den ersten Abflussproben beruhen vor allem auf einer stark von der Löslichkeit dominierten Auswaschung. Diuron besitzt mit $42 \mathrm{mg} / \mathrm{L}$ die höchste Wasserlöslichkeit (Tabelle 1) unter den untersuchten Bioziden und wird am stärksten ausgewaschen, gefolgt von Terbutryn und Cybutryn. Bemerkenswert ist, dass trotz der unterschiedlichen Wasserlöslichkeiten von Cybutryn $(7 \mathrm{mg} / \mathrm{L})$ und Terbutryn $(25 \mathrm{mg} / \mathrm{L})$ die Auswaschraten sehr ähnlich sind. Wäre ausschließlich die Wasserlöslichkeit austragsrelevant, so sollten tendenziell eher für Cybutryn und Carbendazim (Wasserlöslichkeit $8 \mathrm{mg} / \mathrm{L}$ ) ähnliche Konzentrationsniveaus auftreten. Die Konzentrationen von Carbendazim liegen über die Bewitterungsdauer aber um einen Faktor von 1,5 bis 2 tiefer. Daraus lässt sich schließen, dass

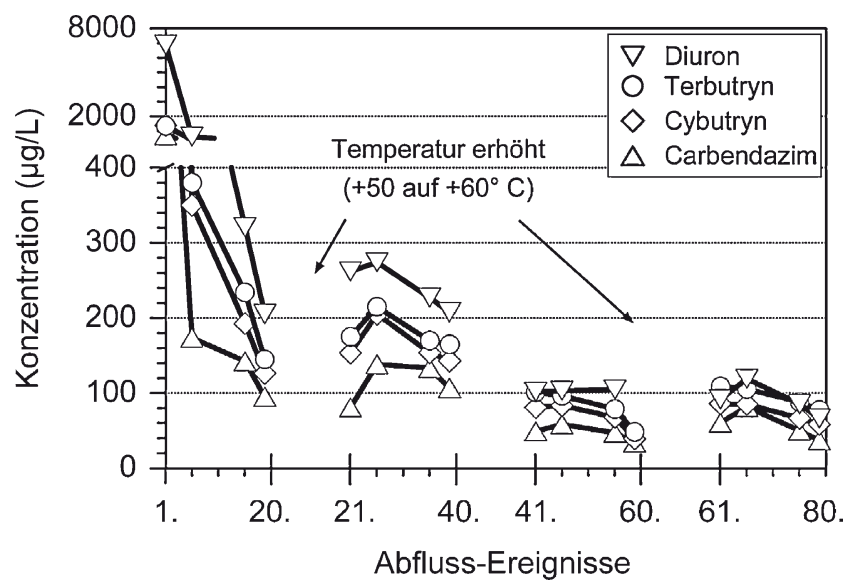

Abb. 3 Auswaschung von vier Bioziden aus kunstharzgebundenem Putz während 80 Abfluss-Ereignissen. Der Abfluss wurde durch 80 Beregnungsintervalle mit jeweils $85 \mathrm{~mm}$ Schlagregen in $60 \mathrm{~min}$ verursacht. Vier Phasen mit jeweils 20 Intervallen $(=5$ Tage) und nachfolgenden zwei Ruhetagen wurden untersucht. Vor der 21. und 61. Beregnung wurde die Temperatur jeweils von $+50^{\circ} \mathrm{C}$ auf $+60^{\circ} \mathrm{C}$ erhöht die Wasserlöslichkeit der Biozide nicht alleine für die Auswaschung bestimmend ist, sondern noch andere Prozesse wie Photoabbau, Verflüchtigung und Bindungsvermögen in der Putzmatrix berücksichtigt werden müssen (Paulus 2004). Zum effektiven Einfluss dieser Prozesse liegen aber noch keine gesicherten Kenntnisse vor. Diese ersten experimentellen Daten verdeutlichen, dass Fassadenabwasser entweder den gebäudenahen Boden belasten kann oder im Falle einer Trennkanalisation bis in den Vorfluter durch unbelastetes Regenwasser erheblich verdünnt werden muss. Untersuchungen von Lindner (1997) und Menge (2005) bestätigen die hohe Auswaschdynamik aus Farbsystemen und die resultierende Belastung des abfließenden Regenwassers.

Abbildung 3 zeigt darüber hinaus, dass eine Temperaturerhöhnung von $+50{ }^{\circ} \mathrm{C}$ auf $+60^{\circ} \mathrm{C}$ die Freisetzung der Biozide erhöht (Luftfeuchtigkeit nahe 90\%). Die temperaturabhängige Diffusion dürfte hier eine entscheidende Rolle bei der Nachlieferung von Wirkstoffen an die Fassadenoberfläche spielen. Vor allem in gealterten Fassaden, in denen die Oberflächen bereits an Bioziden verarmt sind, bestimmen diffusive Prozesse die Stoffanreicherung an der Fassadenoberfläche und damit die für die Auswaschung verfügbaren Stoffmengen.

Im ersten Fassadenabflussereignis mit $85 \mathrm{~mm}$ Beregnungsmenge in 60 min wurden durchschnittlich 1,5\% der zugesetzten Menge von Diuron $\left(1500 \mathrm{mg} / \mathrm{m}^{2}\right)$ ausgewaschen. Die hohe Fracht verringerte sich entsprechend der Konzentrationsabnahme nach rund $5100 \mathrm{~mm}$ Beregnung rasch auf beispielsweise $0,1 \%$ im 61 . Abflussereignis. Bezogen auf die gesamte Bewitterungsdauer sind aus dem kunstharzgebundenen Putz von der Gesamtmenge 7\% Carbendazim, 19\% Cybutryn, $21 \%$ Terbutryn und 29\% Diuron im Fassadenabfluss wiedergefunden worden.

Die Konzentrationen der Biozide nehmen nicht nur über die gesamte Versuchsdauer exponentiell ab, sondern ebenfalls über jede einzelne Beregnung im Bereich von einer Größenordnung. Abbildung 4 zeigt den Konzentrations-
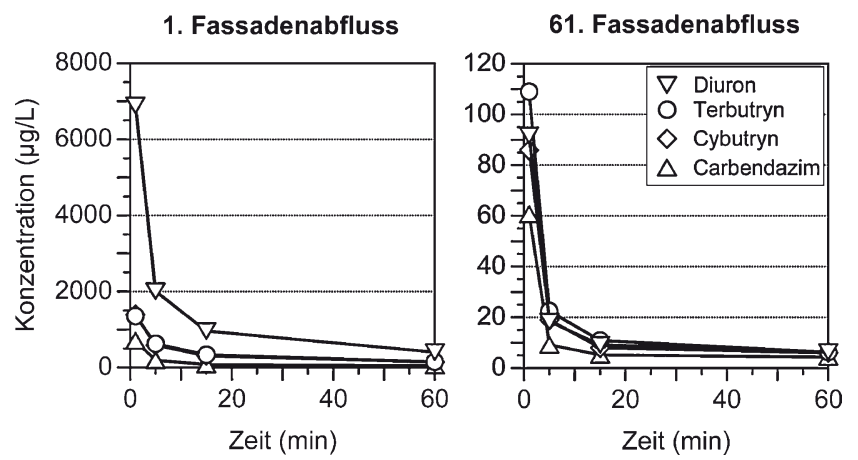

Abb. 4 Auswaschung von Diuron, Terbutryn, Cybutryn und Carbendazim aus Fassadenputz während der 1. und 61. Beregnung mit jeweils 60 min Beregnungsdauer 
verlauf über 60 min während der 1. und 61. Beregnung beispielhaft für Cybutryn, Terbutryn, Diuron und Carbendazim. Unter Berücksichtigung der Beregnungsmenge wurden 50 bis $60 \%$ der Biozidfracht eines Ereignisses bereits innerhalb der ersten 15 Minuten abgewaschen. Ergänzende Laborversuche mit kleineren Prüfkörpern haben die abnehmenden Auswaschraten, den Einfluss der Wasserlöslichkeit auf die Auswaschung der Wirkstoffe und Diffusionsprozessen bestätigt. Übertragen auf die reale Situation an Gebäuden lässt sich ableiten, dass auch kurze Regenereignisse von großer Relevanz sind - dies insbesondere im Sommer unter dem Einfluss von höheren Temperaturen.

\subsection{Abschätzung der Gewässerbelastung am Beispiel Cybutryn}

Abbildung 5 zeigt den erwarteten, d. h. modellierten Konzentrationsverlauf von Cybutryn über vier Monate nach Fertigstellung bzw. Bewitterung der sieben neuen Gebäudefassaden. Der pulsartige Verlauf lässt rund 20 Überschreitungen des akuten Qualitätsziels $\mathrm{HC} 5_{\mathrm{EC} 50}$ von $110 \mathrm{ng} / \mathrm{L}$ Cybutryn erwarten, die im betrachteten Zeitraum rund $120 \mathrm{~h}$ andauern. Einzelne Regenereignisse, die an Fassaden abflusswirksam auftreten, führen zur Überschreitung des akuten Wasserqualitätskriteriums. Mit zunehmender Bewitterung reduziert sich aber das Belastungsrisiko. Das Vorkommen hängt demnach stark vom verfügbaren Stofflager ab, welches an bewitterten Fassaden gleichzusetzen sein dürfte mit dem Gebäudealter. Wie das Konzentrationsniveau für Cybutryn
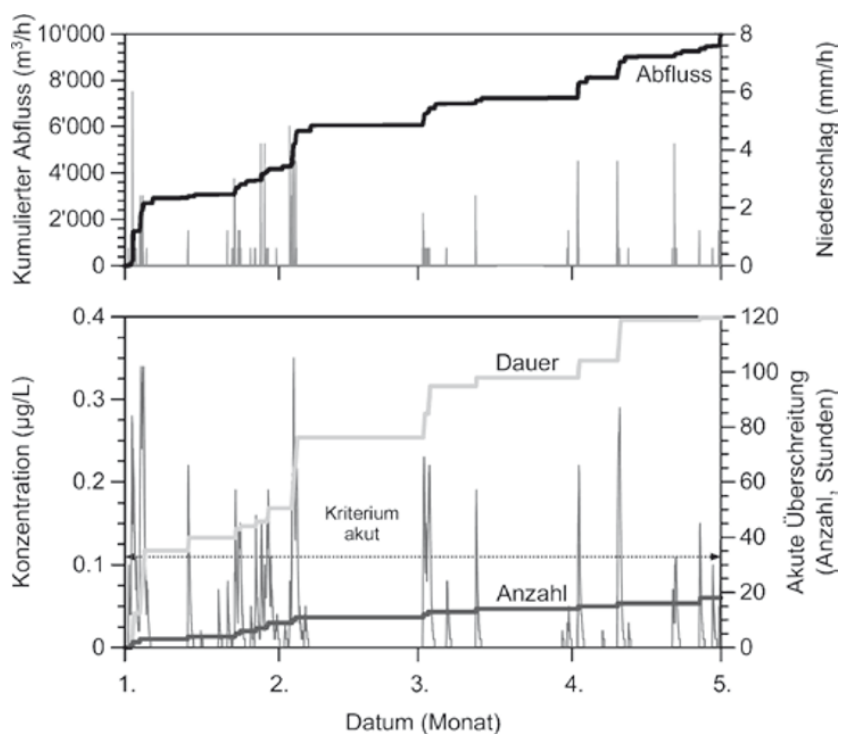

Abb. 5 Modellierung des Vorkommens von Cybutryn im Vorfluter und die Anzahl und Dauer der Überschreitungen des akuten Kriteriums $\mathrm{HC} 5_{\mathrm{EC} 50}$ von $110 \mathrm{ng} / \mathrm{L}$. Dargestellt ist der Zeitraum Januar bis Mai 2003. Die Modellierung basiert auf einem Szenario, welches bei Walser et al. (2008) detaillierter beschrieben ist im Laborversuch vermuten ließ, ist selbst in einem großen Einzugsgebiet eine hinreichend starke Verdünnung des belasteten Fassadenwassers nicht immer zu erwarten. Allemal werden die kleinen Gewässer die höchsten Konzentrationen aufweisen. Wird ein weniger ökotoxisches Biozid unter den gleichen Randbedingungen eingesetzt und ausgewaschen, beispielsweise Terbutryn, so lässt sich in jedem Fall das akute Belastungsrisiko reduzieren. Weitere Anhaltspunkte geben Walser et al. (2008). Ökotoxikologen wiederum beginnen erst, die Wirkung von kurzzeitig auftretenden Expositionen für aquatische Organismen abzuschätzen (Vallotton et al. 2008) und in dynamischen Modellen zu integrieren (Ashauer et al. 2007).

In der Fassadenbeschichtung, dem Boden und im Gewässer können Biozide mikrobiologisch und photolytisch abgebaut werden. Nur unter Berücksichtigung von den wesentlichen Abbauprodukten lässt sich das Belastungsrisiko in Gewässern und Boden umfassend bewerten. Für die wenigsten Wirkstoffe sind aber die Abbauvorgänge, insbesondere die Abbauprodukte, bekannt. Von Cybutryn ist der relativ persistente Metabolit M1 bekannt. Im Laborversuch wurde M1 nur unter Einfluss von UV-Bestrahlung im Fassadenwasser nachgewiesen. Angesichts der angedeuteten Ökotoxizität (Abb. 6), welche auch nach Schmidt et al. (2007) höher als bei Terbutryn und Atrazin liegen dürfte, sollte M1 besondere Aufmerksamkeit geschenkt werden. Für die Ableitung einer SSD bzw. von Wasserqualitätskriterien reichte die Datenbasis aber nicht aus. Hierzu sind zusätzliche experimentelle und modellbasierte Abklärungen angezeigt.

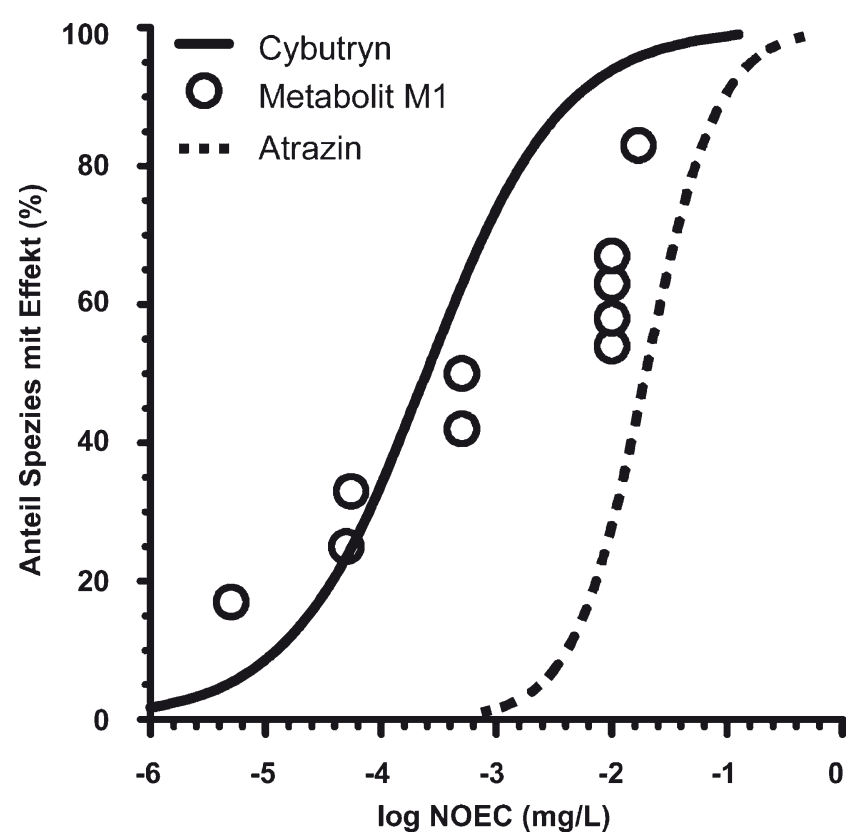

Abb. 6 Spezies-Sensitivitätsverteilungen für NOEC-Daten von Cybutryn und Atrazin sowie NOEC-Daten für den Metabolit M1, welcher aus Cybutryn gebildet wird 


\section{Schlussfolgerungen}

Die heute gebräuchlichen Biozide in Fassadenbeschichtungen weisen ein hohes ökotoxikologisches Wirkungspotential auf. Nicht alle Wirkstoffe gelten aber als persistent oder bioakkumulativ. Insbesondere die persistenten Biozide werden mit dem Fassadenabfluss in die Umwelt eingetragen. Verschmutztes Fassadenabwasser kann diffus im Boden versickern sowie im Regenkanal von Trennkanalisationen fast ohne Retardation und Abbau oder bei Regenwetter unter Umgehung der Kläranlage direkt ins Gewässer transportiert werden (Regenwasserentlastung). Auch in Kläranlagen werden einige der Biozide kaum eliminiert. Ob technische Barrierensysteme, wie künstliche Bodenpassagen und Sickermulden, auch organische Spurenstoffe effizient zurückhalten, ist bisher nicht untersucht worden. Es ist insofern unklar, wie Boden und Regenwasser nachhaltig geschützt werden können, wenn dass Fassadenabwasser teilweise hoch belastet ist. Die beobachteten Konzentrationen und Frachten im Fassadenabfluss und Regenwasserabfluss lassen in jedem Fall den Schluss zu, dass bei der urbanen Regenwasserentsorgung auch Wasserqualitätsaspekte verstärkt Beachtung finden müssen.

Die Zusammenführung von Expositionsdaten und wirkungsbasierten Qualitätskriterien ermöglicht die Identifikation der wichtigsten austrags- und belastungsrelevanten Randbedingungen wie Baualterstruktur, Biozidmenge, Schlagregenintensität und Temperatur. Abschätzungen mittels definierter Austragsszenarien ermöglichen, potenzielle Belastungsschwerpunkte frühzeitig zu identifizieren und damit Umweltuntersuchungen zielgerichteter durchzuführen.

Aus den derzeit verfügbaren Laborergebnissen zur Auswaschung von Bioziden kann abgeleitet werden, dass mit zunehmendem Alter der Fassaden die Abflusskonzentrationen sinken. Die rasche Verarmung an Bioziden in Fassadenbeschichtungen wirft die Frage auf, wie nachhaltig chemischer Fassadenschutz ist. Die durch den Klimawandel hervorgerufenen vermehrt auftretenden sommerlichen Starkregenereignisse können die Auswaschung noch beschleunigen. Höhere Temperaturen durch die globale Erwärmung ermöglichen ein besseres Wachstum der Algen und Pilze, so dass gegebenenfalls in der Zukunft auch die eingesetzte Biozidmenge pro Fläche steigt. Daraus würde eine höhere freisetzbare Menge resultieren. Durch die zunehmende Wärmedämmung von Gebäuden ist zudem damit zu rechnen, dass der Biozideinsatz in Fassadenbeschichtungen bezogen auf die Anzahl der Gebäude bzw. Fassadenflächen noch zunehmen wird (Burkhardt et al. 2006). Die gegenwärtige Baupraxis weist hier in eine eindeutig erkennbare Richtung.

\section{Empfehlungen und Ausblick}

Für einen ökologisch nachhaltigen Boden- und Gewässerschutz bieten sich dezentrale Maßnahmen an der Quelle an.
Diese sollten proaktiv und interdisziplinär mit Herstellern sowie Mitarbeitern der Material- und Umweltwissenschaften sowie der Siedlungswasserwirtschaft entwickelt werden. Zur Materialoptimierung können beispielsweise innovative Einbettungsverfahren für Wirkstoffe, die Anwendung weniger problematischer Wirkstoffe und die Entwicklung biozidfreier Beschichtungen zählen. Geeignete Barrierensysteme zur Retention von belasteten Abflüssen und eine nachhaltigere Architektur (konstruktiver Feuchteschutz) sollten ebenfalls geprüft werden. Ein ganzheitliches Management von Baumaterialien und Regenwasserbewirtschaftung ist für Siedlungsräume gefordert.

In Baumaterialien eingesetzte Wirkstoffe werden pulsartig mit dem Regenwasser in Boden und Gewässer eingetragen. Die ökoxikologischen Effekte der dynamischen Belastung auf verschiedene Umweltkompartimente bedürfen einerseits weiterer experimenteller Abklärungen, andererseits sollten Expositions-Effekt-Modelle entwickelt werden, die akute und chronische Belastungsrisiken vorhersagen können. Ergänzend durch systemübergreifende Stoffflussanalysen können die Stoffströme bilanziert und die Belastungsschwerpunkte abgeschätzt werden.

Die Biozidproduktenrichtlinie 98/8/EG der EU (Europäische Union 1988) bzw. die Dokumente zur wirkstoffspezifischen Risikoabschätzung weisen in Bezug auf Materialschutzmittel für Fassaden eine Bewertungslücke auf. Der direkte Eintragspfad ins abfliessende Regenwasser wird in keinem Expositionsszenario beurteilt. Hier wäre eine Nachbesserung wünschenswert, denn der oberflächliche Abfluss im Gebäudebereich sowie die Einleitung von Fassadenwasser ins Drainagesystem sind weit verbreitete Entsorgungswege. Insbesondere bei der Regenwassertrennkanalisation wird das belastete Regenabwasser meistens direkt in die Gewässer eingeleitet.

Im Rahmen des Projekts URBIC (www.eawag.ch/urbic) werden derzeit Untersuchungen im Labor, im halbtechnischen Maßstab und in einem Siedlungsgebiet unter natürlichem Witterungseinfluss durchgeführt. Ziel der Studien ist, Transportprozesse von Bioziden aus Materialschutzanwendungen zu quantifizieren und für einen nachhaltigen Schutz der Regenabwasser- und Gewässerqualität technische Maßnahmen abzuleiten.

Danksagung Die Arbeit wurde mit großer Unterstützung von Bastian Etter, Michael Federer, Jan Landert, Thomas Marti und Adrian Wichser sowie mit Hilfe anderer Personen durchgeführt. Wir möchten unseren Kollegen von Eawag und Empa ganz herzlich danken. Das Projekt URBIC wurden durch das Bundesamt für Umwelt (BAFU), Bern, und das Amt für Abfall, Wasser, Energie und Luft (AWEL), Zürich, gefördert.

Zusatzinformation Die Datengrundlage für die Ableitung von ökotoxikologischen Qualitätskriterien ist im Online Appendix (Electronic Supplementary Material) aufgeführt. Für jeden Wirkstoff sind die chronischen und akuten Daten verfügbar. 


\section{Literatur}

Arrhenius A, Backhaus T, Gronvall F, Junghans M, Scholze M, Blanck H (2006) Effects of three antifouling agents on algal communities and algal reproduction: mixture toxicity studies with TBT, Irgarol, and Sea-Nine. Archives of Environmental Contamination and Toxicology 50:335-345

Ashauer R, Boxall ABA, Brown CD (2007) New ecotoxicological model to simulate survival of aquatic invertebrates after exposure to fluctuating and sequential pulses of pesticides. Environ Sci Technol 41:1480-1486

Balsiger C, Niederhauser P, Jäggi O, Meier W (2007) Gewässerbelastung durch Pestizide. GWA Gas, Wasser, Abwasser 3:177-185

Bürgi D, Knechtenhofer L, Meier I, Giger W (2008) Priorisierung von bioziden Wirkstoffen. Umweltwiss Schadst Forsch (10.1007/ s12302-008-0032-2)

Burkhardt M, Kupper T, Rossi L, Boller M (2006) Einsatz von Bioziden in Fassaden. Applica 12:2-8

Burkhardt M, Kupper T, Hean S, Haag R, Schmid P, Kohler M, Boller M (2007) Biocides used in building materials and their leaching behavior to sewer systems. Water Sci Technol 56:63-67

European Committee for Standardization (2006) Indicative list of regulated dangerous substances possibly associated with construction products under the CPD. CEN/TC 351, European Commission, Brüssel. http://www.umweltbundesamt.de/bauprodukte/ dokumente/list-cpd.pdf (15.12.2008)

Chèvre N, Loepfe C, Singer H, Stamm C, Fenner K, Escher B (2006) Including mixtures in the determination of water quality criteria for herbicides in surface water. Environ Sci Technol 40:426-435

Dahl B, Blanck H (1996) Toxic effects of the antifouling agent Irgarol 1051 on periphyton communities in coastal water microcosmos. Marine Poll Bull 32:342-350

EOTA (2000) ETAG 004 - Guideline for European Technical Approval of External Thermal Insulation Composite Systems with Rendering. Edition March 2000, European Organisation for Technical Approvals, Brüssel. http://www.eota.be/pdf/FG004 ETICS_FIN2.pdf (15.12.2008)

Eriksson E, Baun A, Scholes L, Ledin A, Ahlman A, Revitt M, Noutsopoulos C, Mikkelsen PS (2007) Selected stormwater priority pollutants - a European perspective. Sci Total Environ 383:41-51

European Chemicals Bureau (2003) Technical Guidance Document on Risk Assessment. European Commission, Joint Research Centre, Ispra. http://ecb.jrc.ec.europa.eu/documents/TECHNICAL GUIDANCE DOCUMENT/EDITION 2/tgdpart2 2ed. pdf $(15.12 .2008)$

Europäische Union (1988) Richtlinie des Rates vom 21.12.1988 zur Angleichung der Rechts- und Verwaltungsvorschriften der Mitgliedsstaaten über Bauprodukte 89/106/EWG (BPR). http:// eur-lex.europa.eu/LexUriServ/LexUriServ.do?uri=CELEX: 31989L0106:DE:HTML (15.12.2008)

Europäische Union (1998) Richtlinie 98/8/EG des europäischen Parlaments und des Rates vom 16. Februar 1998 über das Inverkehrbringen von Biozid-Produkten. http://eur-lex.europa.eu/Lex-
UriServ/site/de/oj/1998/1_123/1_12319980424de00010063.pdf (15. 12.2008)

Gerecke A, Müller S, Singer H, Schärer M, Schwarzenbach RP, Sägesser M, Ochsenbein U, Popow P (2001) Pestizide in Oberflächengewässern. Einträge via ARA: Bestandsaufnahme und Reduktionsmöglichkeiten. GWA Gas Wasser Abwasser 81:173-181

Goonetilleke A, Thomas E, Ginnc S, Gilbert D (2005) Understanding the role of land use in urban stormwater quality management. J Environ Manage 74:31-42

Konstantinou IK, Albanis TA (2004) Worldwide occurrence and effects of antifouling paint booster biocides in the aquatic environment: a review. Environ Int 30:235-248

Kupper T, Plagellat C, Brändli RC, de Alencastro LF, Grandjean D, Tarradellas DJ (2006) Fate and removal of polycyclic musks, UV filters and biocides during wastewater treatment. Water Res 40:2603-2612.

Lindner W (1997) Studies on film preservatives: retention of DCMU in outdoor paints. Biofouling 11:179-189

Menge D (2005) Gewässerbelastung durch den Eintrag von Bioziden aus Dachfarben - eine Risikoabschätzung. Merkblätter Band 51, Landesumweltamt Nordrhein-Westfalen. http://www.lanuv.nrw.de/ veroeffentlichungen $/ \mathrm{merkbl} / \mathrm{merk} 51 /$ merk51.pdf (15.12.2008)

Paulus W (2004) Directory of Microbiocides for the Protection of Materials. Springer, Dordrecht

Posthuma L, Suter GW, Traas TP (Hrsg.) (2002) Species Sensitivity Distributions in Ecotoxicology. CRC Press LLC, Boca Raton, Florida

Quednow K, Püttmann W (2007) Monitoring terbutryn pollution in small rivers of Hesse, Germany. J Envion Monit 9:1337-1343

Schmidt R, Berghahn R, Feibicke M, Mailahn W, Meinecke S, Mohr S (2007) Field, fate and effect studies on the biocide N-tert-butylN'-cyclopropyl-6-methylthio-1,3,5-triazine-2,4-diamine (Irgarol). Bericht, Umweltbundesamt, Dessau

Schweizerischer Bundesrat (2005) Verordnung über das Inverkehrbringen von und den Umgang mit Biozidprodukten (Biozidprodukteverordnung, VBP) vom 18. Mai 2005 (Stand am 1. Juli 2008). SR 813.12, Bern. http://www.admin.ch/ch/d/as/2007/851. pdf (15.12.2008)

Steinmann P, Niederhauser P (2008) Pestiziduntersuchungen bei den Hauptmessstellen Furtbach Würenlos und Glatt vor Rhein im Jahr 2007. Bericht, Amt für Abfall, Wasser, Energie und Luft (AWEL), Zürich. http://www.gewaesserqualitaet.zh.ch/internet/bd/awel/gs/ $\mathrm{gq} /$ de/doku/dokumente.html (15.12.2008)

Vallotton N, Eggen RIL, Escher BI, Krayenbühl J, Chèvre N (2008) Effect of pulse herbicidal exposure on scenedesmus vacuolatus: a comparison of two photosynthese II inhibitors. Environ Toxicol Chem 27:1399-1407

Walser A, Burkhardt M, Zuleeg S, Boller M (2008) Gewässerbelastung durch Biozide aus Gebäudefassaden. GWA Gas Wasser Abwasser 8:639-647

Wunder T (2001) Der Einsatz von Algiziden und Fungiziden in Fassadenbeschichtungen. 3. Dahlberg-Kolloqium ,Mikroorganismen und Bauwerksinstandsetzung', Wismar, S. 105-109 (13.-14. September) 